\title{
The Alternative Cellular Energy (ACE) Pathway as a Primary Non-Immunological Defense Mechanism Against Infectious Diseases
}

\author{
W John Martin* \\ Institute of Progressive Medicine, South Pasadena
}

Submission: November 30, 2016; Published: December 01, 2016

*Corresponding author: John Martin W MD, PhD, Institute of Progressive Medicine, 1634 Spruce Street, South Pasadena CA 91030.

Phone: 626-616-2868; E-mail: wjohnmartin@ccid.org

\begin{abstract}
Studies on stealth adapted viruses, which lack the relatively few components normally targeted by the cellular immune system, have helped identify a more basic, non-immunological, cellular defense mechanism against infectious diseases. This protective mechanism is mediated by the alternative cellular energy (ACE) pathway. The ACE pathway comprises an added dynamic (kinetic) quality of the body's fluids that can facilitate various cellular functions, including the suppression of infections. It is proposed that the added kinetic energy is derived from an external force termed KELEA (Kinetic Energy Limiting Electrostatic Attraction). This force apparently loosens the hydrogen bonding between water molecules and may also directly contribute to internal molecular movements within fluids. It is further proposed that the fundamental role of KELEA in Nature is to prevent the fusion and annihilation of electrostatically attracted opposite electrical charges. KELEA is attracted to separated electrical charges and released to nearby water. Parental administration or even drinking of KELEA activated fluids can be used to enhance the body's ACE pathway. Fluctuating electrical activities of the brain and possibly muscles, including the heart, may also function as an antenna for KELEA. Drinking KELEA activated water appears to improve this capacity. Enhancing the ACE pathway has proven to be highly beneficial in the therapy of various infectious illnesses. As outlined in this paper, the ACE pathway has several major advantages when compared to the immune system as a primary defense mechanism against various infectious diseases.
\end{abstract}

Keywords: Energy Medicine; Alternative Cellular Energy; Enerceutical; Waterceutical; Stealth Adapted Viruses; Kinetic Energy Limiting Electrostatic Attraction, Immunity; HANSI, Enercel; Homeopathy; Energy Medicin; Enerceutical; Waterceutical; Stealth Adapted Viruses; HIV; CMV; HSV; HZV; Zika

Abbreviations: ACE: Alternative Cellular Energy; KELEA: Kinetic Energy Limiting Electrostatic Attraction; HIV: Immunodeficiency Virus; HCMV: Human Cytomegalovirus; CAM: Complementary and Alternative Medicine; CFS: Chronic Fatigue Syndrome; CPE: Cytopathic Effect; CTL: cytotoxic T Lymphocyte; ICE: Insufficient Cellular Energy

\section{Introduction}

The immune system is typically viewed as comprising a more primitive innate inflammatory reaction that can be selectively targeted and amplified by an adaptive immune response [1]. The long-lasting adaptive response is evoked by prior exposure to each specific pathogen and is mediated by both antibody producing B lymphocytes and by $\mathrm{T}$ lymphocytes able to selectively kill abnormal cells and to secrete various cytokines. Immunization refers to the use of more purified antigens as a means of eliciting an adaptive immune response before exposure to the actual pathogen. It is typically more effective in generating $\mathrm{B}$ cell mediated rather than $\mathrm{T}$ cell mediated immunity. This is particularly relevant to the protection against most viruses, since cytotoxic T lymphocytes (CTL) play the predominant role in anti-virus immunity [2].
The Clonal Selection Theory of Immunology is based on the finding that individual lymphocytes are genetically programmed to recognize only a single antigenic specificity [3]. For effective engagement of CTL's with a virus infected cell, it is necessary that the infected cell express multiple copies of relatively few virus antigens, rather than low numbers of a plethora of different antigens. A striking example of this restriction is seen with human cytomegalovirus (HCMV). Although the virus codes for over a hundred different proteins, the vast majority of the evoked CTL's collectively target only three virus antigens $[4,5]$. Through deletion or mutation of the genes coding for these three proteins, it is possible for HCMV to essentially avoid effective recognition by the cellular immune system. This immune evasion mechanism is termed stealth adaptation $[6,7]$. 
It was initially shown as occurring with an African green monkey simian cytomegalovirus (SCMV) [8], but can potentially occur with all cell damaging (cytopathic) viruses [9].

The brain is particularly susceptible to symptomatic illness caused by limited localized cellular damage, as can occur with stealth adapted virus infections. This is because of the spatial specialization of functional activities of the brain. Stealth adapted viruses have been repeatedly cultured from the blood and cerebrospinal fluid (CSF) of patients with a wide range of common neurological and psychiatric illnesses [10-17]. These include autism and behavioral problems in children; chronic fatigue syndrome (CFS), depression and psychosis in adults; and Alzheimer's disease in the elderly. Stealth adapted viruses can similarly induce acute neurological disease when inoculated into cats [18]. As expected, in neither human nor animal stealth adapted virus infections is there inflammation within the brain. $[11,15,18]$. Yet, virus infected patients do not typically experience progressive illnesses and the virus inoculated cats clinically recover from the acute illness. These observations indicate a potential non-immunological process of virus suppression.

A similar conclusion was reached during the in vitro culturing of stealth adapted viruses. In routine virus cultures, what appears at first to be the beginning of a typical cytopathic effect (CPE) can resolve such that the cells regain their normal appearance. Refeeding the cultures with fresh medium leads to reactivation of the initial CPE [19]. Frequent refeeding of the cultures promotes the further development of the CPE, such that the affected cells display marked foamy, vacuolated appearances with considerable cell death and commonly with syncytia formation [6]. Considerable recovery again occurs if the cultures are no longer refed with fresh medium. This recovery correlates with the production of particulate materials with energy transducing capacities [19]. These materials are typically pigmented, fluorescent, electrostatic, occasionally ferromagnetic and have electron donating, lipid synthesizing and water activating activities. The latter activity can be observed as the formation of vapor bubbles when the particles are placed into water. Individual particles form as self-assembled aggregates and as longer fibers and threads. Gas chromatography - mass spectroscopy (GC-MS) and energy dispersive X-ray (EDX) analyses indicated that the particles comprise a range of aliphatic and aromatic chemical entities with selective mineral binding properties (19, unpublished). Adding a few particles to fresh tissue culture medium is strikingly effective in suppressing the reactivation of the CPE that normally follows refeeding of virus infected cultures [19].

A feature of both in vivo and in vitro stealth adapted virus infected cells is the marked disruption of the cells' mitochondria (the main source of energy from the metabolism of food) $[11,20]$. The survival in these cells was attributed to the energy transducing particulate materials, which were accordingly termed alternative cellular energy (ACE) pigments. Inhibition of reactivation in repaired stealth adapted virus cultures was also achieved using small amounts of a supposedly homeopathic remedy termed HANSI (Homeopathic Activator of the Natural System Immune). The demonstration of anti-virus activity of HANSI in tissue cultures clearly excluded a direct role of the immune system. Based on the ACE pathway concept, the United States manufacturer of HANSI renamed the product to Enercel.

In spite of being termed homeopathic, GC-MS analysis of HANSI and Enercel showed that they contained detectable levels of Lidocaine, a dipolar compound. Further studies on virus culture-derived ACE pigments and ACE pigments directly obtained from stealth adapted virus infected patients, led to an understanding that many dipolar chemicals are able to alter the physical properties of water and other fluids. The changes in water include reduction in surface tension and increased internal dynamic (kinetic) activity. The latter can be seen as changes in the dissolving patterns of particles of neutral red dye lightly sprinkled onto the surface of the water. The particles can remain stationary when sprinkled onto non-active water and slowly become surrounded by an expanding circular patch of dye. In contrast and to a varying extent, neutral red dye particles sprinkled onto activated water may sink below the surface. The dissolving particles will form long streaks resulting from to-and-fro movements. Clusters of non-dissolved particles may also form in which individual particles are occasionally rapidly rejected, only to slowly return towards the cluster and often to be repelled again. A more quantitative measurement of water activation is its volatility as assessed as the loss of weight of water in closed but not completely sealed containers. Weight losses in closed containers of activated water can exceed 0.5 $\mathrm{mg} / \mathrm{ml}$ within several hours, with control values being $<0.1 \mathrm{mg} /$ $\mathrm{ml}$ even at 24 hours [21-23]. The increased volatility and other biophysical changes in activated water are consistent with a reduction in hydrogen bonding between water molecules [24].

\section{Methods of Activating Water}

The water from certain worldwide locations is naturally more active than regular water, presumably from being within an environment with increased KELEA. Higher than normal levels of KELEA can also be achieved using several procedures. These include the rapid on-off switching of devices, which acquire separated electrical charges. The attracted KELEA is released upon the repetitive switching-off of the device, allowing for KELEA to spread to nearby water. Ordinary water does not directly absorb KELEA from the environment, but the increased levels of KELEA created by the on-off electrical switching can achieve water activation. Once water is sufficiently activated, its separated electrical charges created by the loosened hydrogen bonding can allow for the direct attraction of KELEA from normal environments. This can result in additional activation, as well as in the activation of added water. This principle explains the 
retention of activity with the serial dilutions used in homeopathy.

Examples of electrical switching devices include those based upon the work of Edgar Cayce (Violet Ray); Royal Raymond Rife (Beam Ray); Georges Lakhovsky (Multi-Wave Oscillator) and Panos Pappas (Papimi; Pulsed Electromagnetic Field) (Reviewed in [25]). Far simpler methods are available, including the on-off connection of metals with differing intrinsic electrochemical potentials. In a different approach, opposite electrical charges can be repetitively directed towards each other and, thereby, increase the attraction of KELEA for release into nearby water. This can explain the usefulness of converging, interrupted light sources, such as the Energy Enhancement System of Dr. Sandra Rose Michael or more simply by using opposing strobe lighting [26]. A similar effect can be achieved with direct electrical currents proceeding in opposing directions, as in a bifilar coil [27]. KELEA levels are also increased within spaces lined by alternating electrical conducting and insulating materials, as employed by Dr. Wilhelm Reich in his "orgone energy" chambers [28]. Certain pyramids and crystals can also influence the natural flow of KELEA.

Various dipolar compounds can also attract and release KELEA possibly in an oscillatory manner. Many such compounds are listed in earlier publications and include mineral rich compounds such as humic and fulvic acids, zeolites, mica, ground and heated volcanic rocks, magnesium oxide pellets, terpenes and many ceramics. Other groupings of dipolar compounds include certain pharmaceuticals; several foods termed Enerceuticals ${ }^{\mathrm{TM}}$, which include various herbal tinctures; different gases; and even previously activated fluids.

The body can also produce water activating materials in the form of ACE pigments. Their production is seen in patients with insufficient cellular energy (ICE). ACE pigments can be seen in live cell microscopy of blood samples as vibrating fibers and moving particles. They can also be tentatively identified in tissues using periodic acid Schiff (PAS) and other staining methods. ACE pigment particles may also be seen in dried perspiration and attached to hair. The UV fluorescence of ACE particles is significantly enhanced by staining with certain dyes, including neutral red. In some patients, the electrostatic property of ACE particles leads to the unfortunate diagnosis of delusional parasitosis [29]. A better, but still inappropriate diagnostic term is Morgellon's disease [30].

An intriguing suggestion supported by several observations is that some aspects of the fluctuating electrical activities of the brain and possibly muscles, including the heart, may provide a more basic, natural means for the body to attract KELEA and to enhance the body's ACE pathway [31]. A supporting observation is the activation of water placed within a room of individuals practicing laughing yoga. The author also knows an individual who is able to activate water, while in a particular state of mind.

\section{Clinical Studies}

Clinical recoveries have commonly been reported using various modalities of Complementary and Alternative Medicine (CAM). The studies typically do not precisely define the efficacy of a single modality or exclude possible psychological influences, commonly referred to as the placebo effect. Since the brain may potentially function as an antenna for KELEA, it is possible that evoked optimism may be acting in concert with a particular therapeutic intervention to increase the body's overall absorption of KELEA. Significant insight into clinical efficacy of CAM has come from studies on Enercel. Traditional homeopathy, as first described by Dr. Samuel Hahnemann, comprises symptom-specific remedies based on the Law of Similars [32]. No published crossover studies have validated the premise of specificity of homeopathic remedies. Indeed, effective remedies previously identified as homeopathy are likely to more simply comprise KELEA activated water. Enercel is such an example, with therapeutic efficacy in a wide range of clinical disorders [33]. These disorders include cancer, infections, infections, neurodegenerative illnesses and wound healing. In an earlier study, tropical diarrhea resolved more rapidly in children receiving two $3 \mathrm{ml}$ injections of Enercel compared to a well matched control group [34]. More recent studies have confirmed the benefit of combined intravenous, intranasal and intra-bronchial administration of Enercel to patients co-infected with the human immunodeficiency virus (HIV) and tuberculosis (TB) [35]. Ongoing studies with Drs. Dariel Laurent and David Christner have shown significant $(>50 \%)$ reductions in HIV loads in AIDS patients receiving Enercel-related drinking water.

ACE pathway based phototherapy achieves marked symptomatic improvements in patients with herpes simplex virus (HSV), herpes zoster virus (HZV) and human papillomavirus (HPV) induced skin lesions [36,37]. ACE pigments within the infected lesions, or in the involved skin in cases of post shingles neuralgia, can be directly activated using neutral red dye in conjunction with ultraviolet-A (UV-A) light illumination. This procedure has been upgraded to UV illumination of neutral red dye dissolved in KELEA activated fluid placed near to, but not in direct contact with the affected skin areas. After relatively short periods of time, the underlying skin areas will also fluoresce if directly illuminated with the UV-A light. A similar approach achieves symptomatic improvement in children with autism, an illness attributed to stealth adapted virus encephalopathy [38].

It has been proposed to Government health officials that KELEA activated water should be immediately evaluated as a potential preventative measure in pregnant women at risk for becoming infected with Zika virus $[39,40]$. Such actions are unlikely to occur if the immune system is perceived as the only natural defense against active virus infections. Progress at the government level will require a greater understanding of the ACE pathway as a basic non-immunological defense mechanism against infectious diseases. 


\section{Advantages of the ACE Pathway}

There are four major and several secondary advantages of the ACE pathway in comparison with the immune system in the defense against infectious diseases. The most decisive is the role of the ACE pathway in suppressing infections due to stealth adapted viruses [6-9]. Based on extensive tissue culture experience, many neurological and psychiatric illnesses have been confidently attributed to ongoing stealth adapted virus infections. These illnesses have not generally been regarded as infectious because of the absence of an accompanying inflammatory response. Major examples include autism and behavioral problems in children, CFS and psychosis in adults and Alzheimer's and other neurodegenerative illnesses in the elderly [10-17].

The range of virus antigens targeted by the cellular immune system is more restricted than the antigens targeted by antivirus antibodies [2]. Anti-virus antibodies can be effective in preventing initial infections with viruses and the blood borne spread of viruses to the central nervous system. Antibodies are far less effective in suppressing ongoing chronic viral infections. Indeed, anti-virus antibodies can exacerbate virus infections through the formation of antigen-antibody complexes that become deposited within blood vessels throughout the body. Part of the pathology of certain stealth adapted virus infections can, accordingly, be attributed to a deleterious effect of the antibody component of the immune system [41].

Stealth adaptation primarily applies to the loss or mutation of the major virus antigens targeted by the cellular immune responses. It is likely that some minor antigens may persist, which can be targeted by CTL, if the immune system is sufficiently stimulated, as may occur during vaccinations. Much of the controversy surrounding the induction of neurological illness by vaccines can be explained by vaccines eliciting cell damaging immunological responses to minor virus antigens expressed on preexisting stealth adapted virus infected brain cells $[42,43]$. In this regard, a preexisting stealth adapted virus infection can provide a valid reason for withholding vaccination, especially with those vaccines that require the use of an adjuvant.

The second major advantage of the ACE pathway is its broad applicability to multiple pathogens, without limitations as to immunologically privileged sites [44]. Evolving antigenic diversity occurring during an infection and within various types of pathogens has also posed difficulties for the body's immunological defenses and for the development of effective vaccines. Especially with infections such as with HIV that directly impair the body's cellular immune system, individuals can become multiply infected with other viruses, bacteria, fungi, protozoa and helminthes. Attempts to immunologically target each type of pathogen is far less practical than enhancing the ACE pathway in the defense against multiple co-infecting pathogens.
Another advantage of the ACE pathway over the immune system in the defense against infections is the lack of scarring as part of the healing process [45]. The immune system attracts inflammatory cells to the sites of infection. Inflammation is followed by the recruitment of fibroblasts with resulting tissue scarring. While scarring is unsightly when it occurs within the skin, it can have major, long-term deleterious effects when it occurs in functional organs, especially the brain. The potential for autoimmunity exists with the immune system, but not with the ACE pathway [46]. This is the fourth major advantage of the ACE pathway compared to the immune system. Allergies are also adverse features of the immune system. For reasons that are not yet fully explained, enhancing the ACE pathway with Enercel, decreases the severity of allergic illnesses, such as asthma and eczema [33].

Among the secondary advantages of the ACE pathway is the positive emotional mindset, which typically develops in those who are experiencing various therapeutic modalities that can enhance the ACE pathway. This is seen, for example, in the monitoring of Quality of Life (QOL) scores in patients receiving Enercel. In contrast, the production of cytokines, as occurs with immune activation, can lead to feelings of exhaustion and fatigue. It is possible that an enhanced ACE pathway can improve the proposed KELEA attracting capacity of the brain, leading to long-term sustainable benefits from temporary intervention with an ACE pathway promoting therapy.

\section{Additional Applications of an Enhanced ACE Pathway}

This review is primarily intended to discuss the ACE pathway in comparison with the immune system. Nevertheless, it is noteworthy that the ACE pathway can be an adjunct to the cellular energy derived from the metabolism of food $[47,48]$. Specifically, enhancing the ACE pathway can potentially alleviate medical conditions in which there are restrictions or limitations on the production of cellular energy from the metabolism of food. These conditions include inadequate oxygen intake, as can occur in chronic obstructive pulmonary disease (COPD); impaired blood supply as in cardiovascular, peripheral vascular and cerebrovascular diseases; inefficient metabolism, as in diabetes; and increased energy demands, including growth and wound healing. Aging may partially reflect a failure of mitochondrial function and may be potentially delayed with an enhanced ACE pathway. Cancer may also reflect a failure of apoptosis due to metabolic disorders, which require heightened cellular energy to correct. If so, the required added energy may potentially be provided by enhancing the ACE pathway [49].

The ACE pathway appears to contribute to improved brain functions in a manner that is not supported by food metabolism. This improvement can be expressed in both improved cognition and other higher level activities. It can also be expressed in the capacity of the brain to maintain homeostasis by adjusting to altered sensory inputs, hormonal levels, etc. This may explain, 
for example, the corrections in blood pressure seen in some patients in response to drinking KELEA activated water. Fluid perfusion within the brain is likely to be increased with KELEA. This is possibly relevant since fluid perfusion is thought to play an important role in the elimination of toxic metabolic byproducts from brain tissues [50]. The most promising aspect of the ACE pathway is the potential positive feedback between the ACE pathway and the proposed antenna function of the brain in attracting KELEA into the body. Short term intensive treatments may, therefore, enhance the brain's proposed longterm capacity to sustain an effective ACE pathway. It is also encouraging to contemplate that certain individuals and even animals may be able to contribute energy to others by simply being in their presence.

\section{Conclusion}

This paper challenges the belief that the immune system is the sole major natural defense against ongoing infectious diseases. It is proposed that a more fundamental process exists that enables the body's cells to outcompete pathogenic microbes. The process is referred to as the alternative cellular energy (ACE) pathway. It presumably results from the absorption of an external energy force termed KELEA (Kinetic Energy Limiting Electrostatic Attraction) and is expressed as a dynamic (kinetic) quality of the body's fluids. Cellular functions, including suppression of infectious diseases, are increased with KELEA. There are several major advantages of the ACE pathway over the immune system, including i) activity against stealth adapted viruses; ii) capacity to respond to multiple co-infections; iii) lack of scarring; and iv) absence of autoimmunity. Various methods of enhancing the ACE pathway are briefly discussed, including the use of KELEA activated water and KELEA energy fields. The ACE pathway is a powerful alternative to the immune system in the control of major infectious diseases.

\section{Acknowledgement}

The Institute of Progressive Medicine is a component of MI Hope Inc., a non-profit public charity.

\section{Conflict of Interest: None}

\section{References}

1. Paul WE (2013) Fundamental Immunology. ( $7^{\text {th }}$ edn), Lippincott Williams \& Wilkins, Philadelphia, USA, pp. 1285.

2. Nathanson $N$ (2007) Viral Pathogenesis and Immunity. $\left(2^{\text {nd }}\right.$ edn) Academic Press, New York, USA, pp. 280.

3. Burnet FM (1959) The Clonal Selection Theory Acquired Immunology. Vanderbilt Univ Press, Nashvill.

4. Wills MR, Carmichael AJ, Mynard K, Jin X, Weekes MP (1996) The human cytotoxic T-lymphocyte (CTL) response to cytomegalovirus is dominated by structural protein pp65: frequency, specificity, and T-cell receptor usage of pp65-specific CTL. J Virol 70(11): 7569-7579.

5. Borysiewicz LK, Hickling JK, Graham S, Sinclair J, Crange MP, et al. (1988) Human cytomegalovirus-specific cytotoxic T-cells, Relative frequency of stage-specific CTL recognizing the $72 \mathrm{kD}$ immediate early protein and glycoprotein $B$ expressed by recombinant vaccinia viruses.
J Exp Med 168(3): 919-931.

6. Martin WJ, Zeng LC, Ahmed K, Roy M (1994) Cytomegalovirus related sequences in an atypical cytopathic virus repeatedly isolated from a patient with the chronic fatigue syndrome. Am J Path 145(2): 440-451.

7. Martin WJ (1994) Stealth viruses as neuropathogens. CAP Today 8(10): 67-70.

8. Martin WJ, Ahmed KN, Zeng LC, Olsen J C, Seward JG, et al. (1995) African green monkey origin of the atypical cytopathic 'stealth virus' isolated from a patient with chronic fatigue syndrome. Clin Diag Virol 4(1): 93-103.

9. Martin WJ (2014) Stealth adaptation of viruses: Review and updated molecular analysis on a Stealth adapted African green monkey simian cytomegalovirus (SCMV). J Hum Virol Retrovirol 1(4): 00020.

10. Martin WJ (1995) Stealth virus isolated from an autistic child. J Autism Dev Disord 25(2): 223-224.

11. Martin WJ (1996) Severe stealth virus encephalopathy following chronic fatigue syndrome like illness: Clinical and histopathological features. Pathobiology 64(1): 1-8.

12. Martin WJ (1996) Simian cytomegalovirus related stealth virus isolated from the cerebrospinal fluid of a patient with bipolar psychosis and acute encephalopathy. Pathobiology 64(2): 64-66.

13. Martin WJ (1997) Detection of RNA sequences in cultures of a stealth virus isolated from the cerebrospinal fluid of a health care worker with chronic fatigue syndrome. Case report. Pathobiology 65(1): 57-60.

14. Martin WJ, Anderson D (1997) Stealth virus epidemic in the Mohave Valley. Initial report of viral isolation. Pathobiology 65(1): 51-56.

15. Martin WJ, Anderson D (1999) Stealth virus epidemic in the Mohave Valley: Severe vacuolating encephalopathy in a child presenting with a behavioral disorder. Exp Mol Pathol 66(1): 19-30.

16. Martin WJ (2015) Stealth adapted viruses - Possible drivers of major neuropsychiatric illnesses including Alzheimer's disease. J Neurol Stroke 2(3): 00057.

17. Martin WJ (2015) Stealth adapted viruses. A bridge between molecular virology and clinical psychiatry. Open J Psychiatry 5(4): 311-319.

18. Martin WJ, Glass RT (1995) Acute encephalopathy induced in cats with a stealth virus isolated from a patient with chronic fatigue syndrome. Pathobiology 63(3): 115-118.

19. Martin WJ (2003) Stealth virus culture pigments: A potential source of cellular energy. Exp Mol Path 74(3): 210-223.

20. Martin WJ (2003) Complex intracellular inclusions in the brain of a child with a stealth virus encephalopathy. Exp Mol Pathol 74(3): 197209.

21. Martin WJ (2015) Therapeutic potential of KELEA activated water. Int J Complementary \& Alternative Medicine 1(1): 00001

22. Martin WJ (2015) Alternative Cellular Energy Pathway Therapy Using KELEA Activated Water. Int J Complementary \& Alternative Medicine $2(2): 00051$.

23. Martin WJ (2015) KELEA Activation of Water and Other Fluids for Health, Agriculture and Industry. Journal of Water Resource and Protection 7(16): 1331-1344.

24. Martin WJ (2015) KELEA: A natural energy that seemingly reduces intermolecular hydrogen bonding in water and other liquids. Open Journal of Biophysics 5(3): 69-79.

25. Martin WJ (2014) Interacting Light Paths Attract KELEA (Kinetic Energy Limiting Electrostatic Attraction) and Can Lead to the Activation of Water. Open Journal of Biophysics 5(4): 321. 
26. Martin WJ (2015) Interacting light paths attract KELEA (kinetic energy limiting electrostatic attraction) and can lead to the activation of water. Open Journal of Biophysics 5(4): 115-121

27. Martin WJ (2015) Interactive electric fields can attract KELEA (kinetic energy limiting electrostatic attraction) and can lead to the activation of water. Int J Complementary \& Alternative Medicine 1(6): 00034.

28. Streik JE (2015) Wilhelm Reich, Biologist. Harvard University Press, Cambridge, MA, pp. 467.

29. Al-Imam AML (2016) A systematic literature review on delusional parasitosis. J Dermatology \& Dermatologic Surgery 20(1): 5-14.

30. Stricker RB, Middelveen MJ (2012) Morgellons disease: More questions than answers. Psychosomatics 53(5): 504-505.

31. Martin WJ (2015) Is the brain an activator of the alternative cellular energy (ACE) pathway? Int J Complementary \& Alternative Medicine 1(1): 00002.

32. Gutman W (1961) The basis of the law of similar. British Homeopathic Journal 50(1): 26-29.

33. Martin WJ, Laurent D (2015) Homeopathy as a misnomer for activation of the alternative cellular energy pathway: Evidence for the therapeutic benefits of Enercel in a diverse range of clinical illnesses. International J Complementary \& Alternative Medicine 2(1): 00045.

34. Izaguire RR, Guzman MR, Fuentes RC, Mena CE, Penate E, et al. (2014) Alternative cellular energy based therapy of childhood diarrhea. In: Martin WJ, Stealth Adapted Viruses; Alternative Cellular Energy (ACE) \& KELEA Activated Water. Author House, Bloomington IN, pp. 103-114.

35. Dubrov V, Dubrova T, Christner D, Laurent D, Martin WJ (2015) Alternative cellular energy based therapy using Enercel $^{\mathrm{TM}}$ in advanced AIDS patients co-infected with tuberculosis and treated in Chernigov, Ukraine. J Hum Virol Retrovirol 2(6): 00061.

36. Martin WJ, Stoneburner J (2005) Symptomatic relief of herpetic skin lesions utilizing an energy based approach to healing. Exp Mol Path 78(2): 131-134.

37. Martin WJ, Stoneburner J (2014) Alternative cellular energy (ACE) pathway activation as the mode of action of neutral red dye phototherapy of human viruses. J Hum Virol Retrovirol 1(4): 00019.

38. Martin WJ (2014) Alternative cellular energy (ACE) pathway activation as natural therapy for autism. In Stealth Adapted Viruses; Alternative
Cellular Energy (ACE) \& KELEA Activated Water. Author House, IN, pp. 87-102.

39. Martin WJ (2016) Enhancing the alternative cellular energy (ACE) pathway in the prevention of Zika virus induced illness. J Human Virol Retrovirol 3(4): 97.

40. Martin WJ (2016) Proposed testing of KELEA activated drinking water in Zika virus infected pregnant women. J Hum VirolRetrovirol 3(5): 104.

41. Martin WJ (1996) Stealth viral encephalopathy: Report of a fatal case complicated by cerebral vasculitis. Pathobiology 64(2): 59-63.

42. Martin WJ (2004) The rationale for vaccines and the potential inadvertent consequences including autism, AIDS and other epidemics. Med Veritas 1: 81-85.

43. Martin WJ (2015) Stealth adaptation of viruses: Implications for therapy and for potential toxicity of vaccines. JOJ Immuno Virology 1(1): 555551.

44. Martin WJ (2016) The ACE pathway in comparison to the immune system in the defense against infectious diseases. J Hum Virol Retrovirol 4(1): 00124.

45. Martin, WJ, Palmer SB (2010) Regenerative wound healing using copper-silver citrate composition. US PatentApplication 20100099758.

46. Shoenfeld Y, Agmon-Levin N, Rose NR (2015) Infection and Autoimmunity. $2^{\text {nd }}(E d n)$ Elsevier Amsterdam, p. 1-4.

47. Martin WJ (2015) Alternative cellular energy. A unifying concept in complementary alternative medicine. Int J Complementary \& Alternative Medicine 1(4): 00022.

48. Martin WJ (2016) Insufficiency of cellular energy (ICE): The basis for many illnesses potentially correctable using KELEA activated water. Int J Complementary \& Alternative Medicine, 4(1): 106.

49. Martin WJ (2016) Cancer as an insufficiency of cellular energy (ICE): Therapeutic approaches based on enhancing the alternative cellular energy (ACE) pathway. Int J Complementary \& Alternative Medicine 3(3): 74

50. Mendelsohn AR, Larrick JW (2013) Sleep facilitates clearance of metabolites from the brain: Glymphatic function in aging and neurodegenerative diseases. Rejuvenation Res 16(6): 518-523.

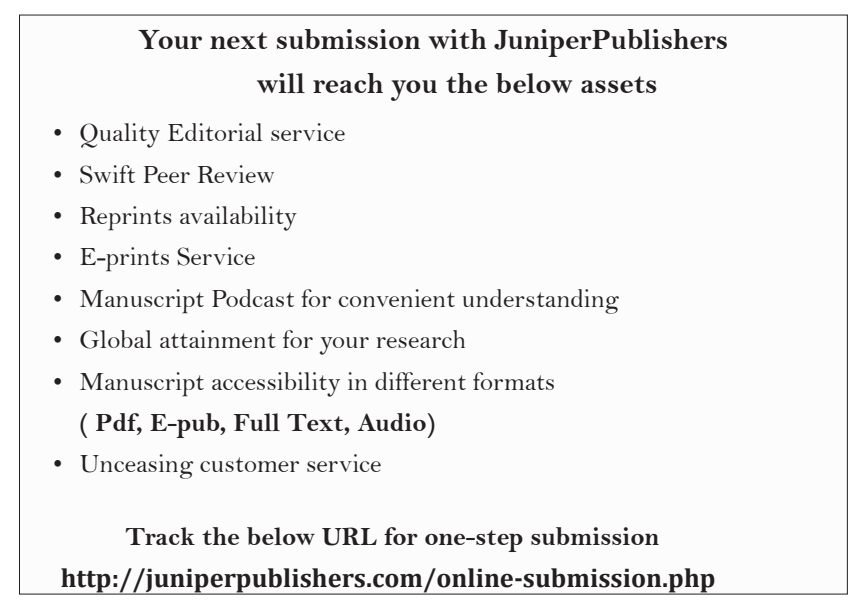

